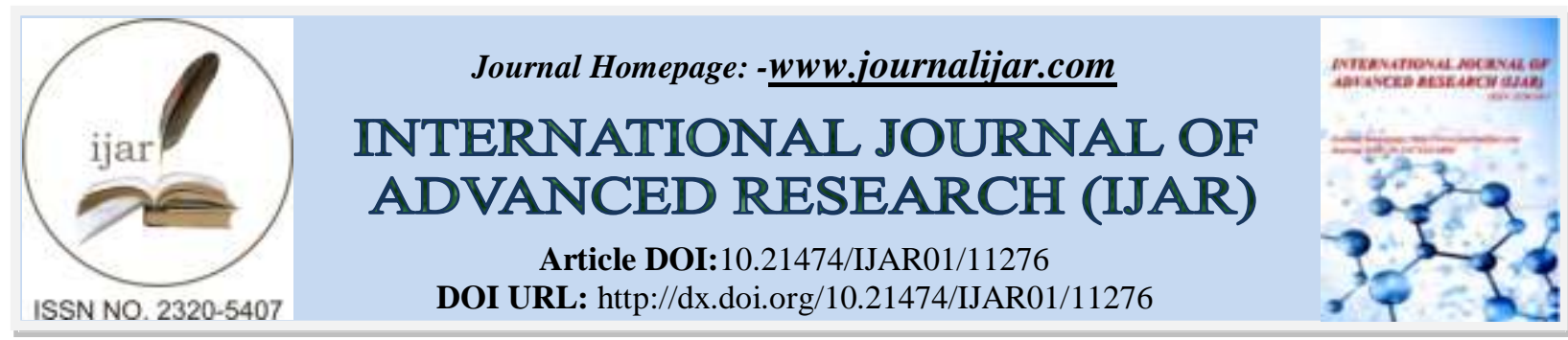

RESEARCH ARTICLE

\title{
MASSIVE HEMOPERITONEUM LAPAROTOMY FOR SAFE SPLEEN DIAGNOSES A RUPTURED OVARIAN CYSTIN WOMAN WITH ANTITHROMBOTIC TREATMENT: CASE REPORT AND LITERATURE REVIEW
}

\author{
Mustapha Ben Moussa, Hicham El. Majdoubi, Marouane Baiss, Ahmed Bounaim, Aziz Zentar and \\ Abdelmounaimait Ali \\ Department of General Surgery, Military Hospital Mohamed V, University Hospital Center Ibn Sina, Rabat,
} Morocco.

\section{Manuscript Info}

\section{Manuscript History}

Received: 05 May 2020

Final Accepted: 10 June 2020

Published: July 2020

Key words:-

Hemoperitoneum, Corpus Luteal Hemorrhage, Anticoagulation, Laparotomy, Safe Spleen

\begin{abstract}
Ruptured corpus luteum cyst is not exceptional finding in women of the reproductive age, but spontaneous massive hemoperitoneum secondary to ruptured corpus luteum cyst in women on anticoagulants is unusual.We hereby report successful management of massive hemoperitoneum wrongly attributed to a splenic lesion. The diagnosis was corrected, in favor of ruptured luteinic cyst, during laparotomy conservative approach. This case report discusses the diagnostic problem and gives a practical example of a trap in front of a spontaneous hemoperitoneum in the context of antithrombotic treatment, and may provides value addition to explorative laparoscopy regarding emerging conservative approach.
\end{abstract}

Copy Right, IJAR, 2020,. All rights reserved.

\section{Introduction:-}

Ovarian hemorrhage with hemoperitoneum is a rare but serious complication of ovulation related to rupture of either the corpus luteum or functional cyst. It is due to treatment using oral indirect anticoagulant and specifically affects young women [1]. Acute pelvic pain in women of childbearing age is a common and frequent cause for admission to emergency room (ER), necessitating emergent medical evaluation especially when it is due to hemoperitoneum[2]. Sometimes it can be difficult to distinguish gynecological from gastrointestinal and urinary tract emergencies because of overlapping symptoms and signs. Various imaging modalities in association with clinical findings play an important role in the characterization of the cause of pain $[3,4]$.

Early diagnosis is necessary to preserve the reproductive systems and the life of the patient in severe cases. Hemoperitoneum may occur in the context of various gynecological emergencies; in some cases it could be a complication of a ruptured hemorrhagic corpus luteum $[4,5]$.

We describe a case of hemoperitoneum from a ruptured hemorrhagic corpus luteum, wrongly attributed to a splenic lesion, in a 20 year-old woman with antithrombotic treatment,was managed efficiently by laparotomy in general surgery operation room.

Corresponding Author: Mustapha Ben Moussa

Address:- Department of General Surgery, Military Hospital Mohamed V, University Hospital Center Ibn Sina, Rabat, Morocco. 


\section{Material and Mathods:-}

A 20 year old nulliparous woman presented to the emergency with acute abdominal pain and vomiting for last three days. There was one episode of metrorrhagia but there was history of anemia and blood transfusion.

She was using oral contraception. She had mitral valve replacement two years back because of severe infectious endocarditis and since then was on acenocoumarol 2-3 mg on alternate days.

General physical examination revealed marked pallor, tachycardia of 130/min and blood pressure of 110/70 mm of $\mathrm{Hg}$.

Abdomen was slightly distended and tender and free fluid was elicitible.

Pelvic examination revealed normal sized uterus. There was no definite mass palpable and the urine pregnancy test was negative.

Laboratory investigations revealed INR $=7$, Hemoglobin $=8.3 \mathrm{gm}$, Platelet count $=222000$ and TLC $=142000$. $\mathrm{CRP}=130$, BHCG negative.

Abdominal CT scan showed massive free fluid perihepatic (figure1), perisplenic and inter intestinal loops, empty uterus.

A provisional diagnosis of spontaneous spleen rupture was made with stable hemodynamic state. Acenocoumarolwas stopped and relayed by unfractionated heparin with APTT and Complete blood count (CBC) monitoring. Antagonization with $10 \mathrm{mg}$ of vitamin K.

The patient was transfused 2 units of blood and 5 units of fresh frozen plasma. Finally, the INR rose to 1,02.

An echo cardiography showed normal functioning valves and computed tomography revealed gross free fluid (figure 1), two potential splenic hypodensity (laceration). No active bleeding vessel was identified on CT Scan C+.

The hemodynamic state deteriored after three days of hospitalization, so decision of explorative laparotomy was made, because of unavailability of the laparoscopic column.

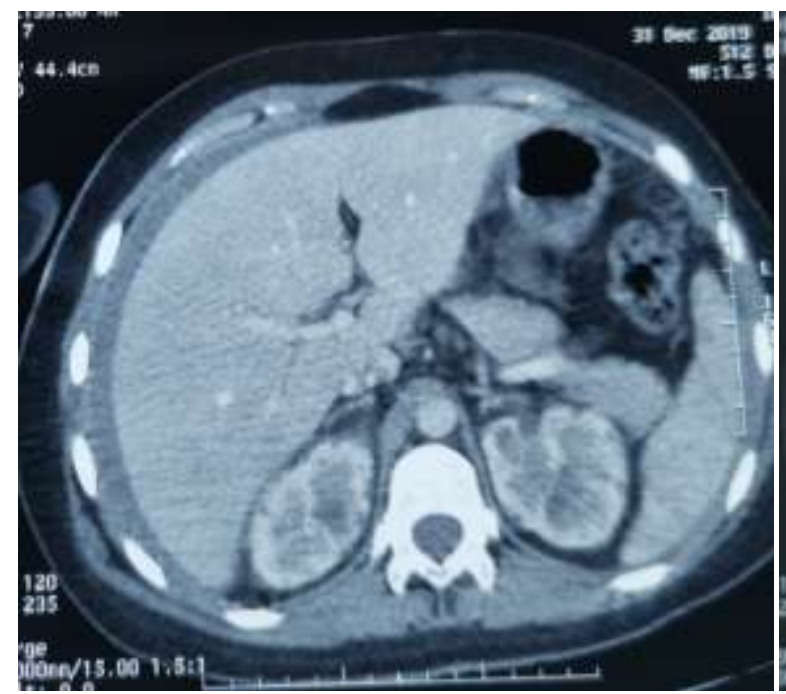

Figure 1:- Axial section of an abdominal CT scan showing perihepatic effusion.

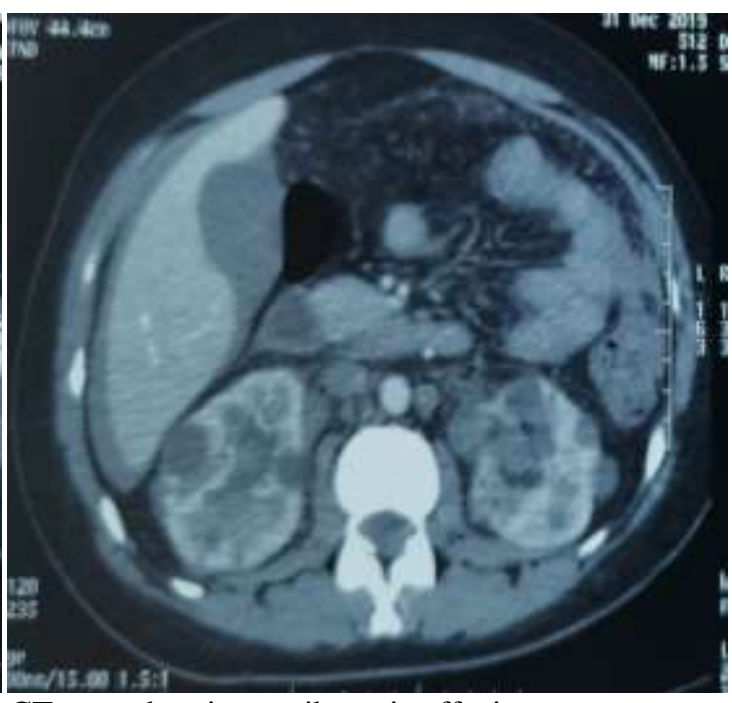

Amidline laparotomy was performed, the exploration objectified a great abundance effusion, which was aspirated. After a warm Saline Serum wash and removal of a large perisplenic clot, the exploration finds an intact spleen. But, surprise, on the pelvis side, presence of anabnormal left ovary, seat of ruptured hemorrhagic cyst with continuous hemorrhagic suffusion (Figure 2). Conservative treatment decision by simple continuous suture of the cystic 
membrane. Hemostasis done, washing and drainage signed, before abdominal wall closure, the end of the intervention.

The post-operative course was uneventful. Discharge on day 3rd after adaptation of the anticoagulant treatment.

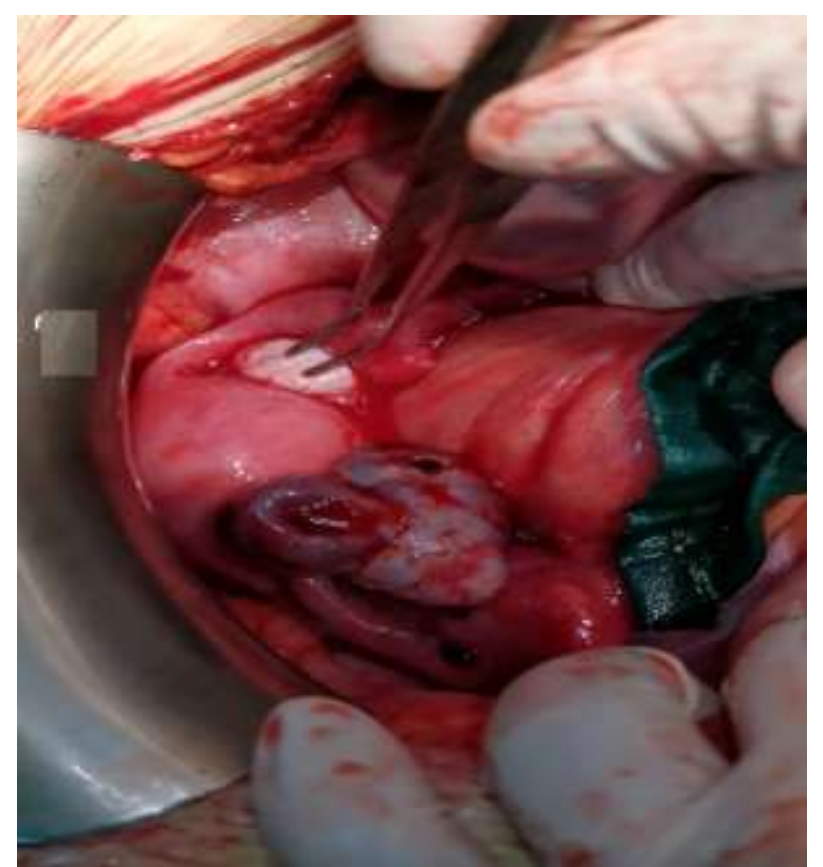

Figure 2:- Per-operative view showing normal right ovary, whereas the left one is seat of a ruptured and oozing hemorrhagic cyst.

\section{Discussion:-}

Corpus luteum cysts are thin-walled, functional vascular structures, and most of them are predisposed to rupture[6]. This rupture, one of the major gynecologic causes of hemoperitoneum, is not known, although it has been suggested that the increased vascularity of the ovary in the luteal phase and pregnancy may predispose to rupture of a corpus luteal cyst. Ongoing bleeding into the cyst can result in rapid enlargement, spontaneous rupture and leakage of blood into the peritoneal cavity [7].

While some haemorrhage associated with ovarian cyst rupture has unclear etiology, there are recognized risk factors. These include abdominal trauma and anticoagulation therapy [8] which is present in our case.

More recent case reports of massive hemoperitoneum from a ruptured corpus luteum cyst are associated with systemic anticoagulation, coagulation disorders, von Willebrand disease, and sickle cell anemia[9][10,11]

According to Hallatt et al. [12] the hemorrhage from a ruptured corpus luteum cyst is likely to be less than in an ectopic pregnancy and likely to be non-recurrent once it stops. Corpus luteal cysts are commonly identified as incidental structures on pelvic ultrasound studies.

Typically at the time of rupture, there may be sharp and sudden onset of pain, which has no typical characteristics. Blood loss can vary from very little bleeding to hypovolemic shock [12].

Hemoperitoneum resulting from ruptured corpus luteum cyst have been described since the early 1900s and is frequently misdiagnosed as ruptured ectopic pregnancy, acute appendicitis, ovarian torsion and endometriosis $[13,14]$ or as spontaneous spleen rupture in our case, imaging influenced by a perisplenic clot and the importance of hemoperitoneum. Also,in one study, the hemoperitoneum was found to be the dominant imaging feature rather than the cyst itself [15] like in our case. 
Observation, pain control and serial hemoglobin monitoring may be appropriate for select cases withhemodynamic stability, but signs of blood loss or hypovolemic shock should prompt immediate laparoscopic intervention.

The diagnosis of ruptured corpus luteal cyst is based on a high historical suspicion (the patient generally is in the luteal phase of the ovarian cycle), clinical features, and laboratory tests. The latter often show anemia, raised CRP, and mild leukocytosis. These signs and symptoms are similar to gastrointestinal tract diseases. Patients may present a wide range of clinical signs, from no signs to severe peritoneal irritation which can be confused with, for example, acute appendicitis. The evaluation of serum $\beta$ hCG-levels is necessary to differentiate ruptured corpus luteal cyst from ruptured ectopic pregnancy, which may have a similar presentation [16].

The differential diagnosis should include ectopic pregnancy, acute trauma, in certain patients; an underlying hematologic condition should be suspected and ruled out. Although most patients require only observation, some need analgesics for pain control and laparoscopy or laparotomy for diagnosis or to achieve hemostasis[7].

In particular, the conservative approach is the first-choice treatment when the patient is hemodynamically stable (systolic BP $>90 \mathrm{mmHg}$ ) with hemoglobin values that keep being constant over 4-6 hours of monitoring [13]. In case of unstable vital signs the patient undergoes surgical treatment. Laparoscopy represents the first minimally invasive approach [14] that can eventually be converted to laparotomy in case of failure or of unstable vital signs such as important hemoglobin decrease over 4-6 hours $(\sim 2 \mathrm{~g} / \mathrm{dL})$ with increasing hemoperitoneum on follow-up imaging studies[17,18].

\section{Conclusion:-}

The etiological diagnosis of a hemoperitoneum is not always easy, especially in emergencies. The hemorrhagic ovarian cyst should be considered, especially in women with antithrombotic treatment and all physicians should be vigilant regadingthis life-threatening complication. Thus, early accurate diagnosis and appropriate therapy reduce significantly morbidity and mortality associated with this condition.

Surgery should be conservative and whenever possible, should include celioscopy. Systematic ovarian blockade should be discussed in women taking long-term oral indirect anticoagulant [1].

\section{Références:-}

1. Crétel E, Cacoub P, Gompel A, Durand JM, Piette JC. [Hemoperitoneum of ovarian origin complicating antivitamin K treatment]. Rev Med Interne. 2000;21(5):428-434.

2. Samraj GPN, Curry RW. Acute pelvic pain: Evaluation and management. Compr Ther. 2004;30(3):173-184.

3. Potter AW, Chandrasekhar CA. US and CT Evaluation of Acute Pelvic Pain of Gynecologic Origin in Nonpregnant Premenopausal Patients. RadioGraphics. 2008;28(6):1645-1659.

4. Kayaba H, Tamura H, Shirayama K, Murata J, Fujiwara Y. Hemorrhagic ovarian cyst in childhood: A case report. J Pediatr Surg. 1996;31(7):978-979.

5. Coulier B, Malbecq S, Brinon P-E, Ramboux A. MDCT diagnosis of ruptured tubal pregnancy with massive hemoperitoneum. Emerg Radiol. 2008;15(3):179-182.

6. Tang LC, Cho HK, Chan SY, Wong VC. Dextropreponderance of corpus luteum rupture. A clinical study. J Reprod Med. 1985;30(10):764-768.

7. Pandit K. Massive Hemoperitoneum from a Ruptured Corpus Luteal Cyst. Juniper Online J Case Stud. 2018;5(5). doi:10.19080/jojcs.2018.05.555672.

8. Gupta N, Dadhwal V, Deka D, Jain SK, Mittal S. Corpus luteum hemorrhage: Rare complication of congenital and acquired coagulation abnormalities. J Obstet Gynaecol Res. 2007;33(3):376-380.

9. Swann RT, Bruce NW. Oxygen consumption, carbon dioxide production and progestagen secretion in the intact ovary of the Day-16 pregnant rat. Reproduction. 1987;80(2):599-605.

10. Hackethal A, Ionesi-Pasacica J, Kreis D, Litzlbauer D, Tinneberg H-R, Oehmke F. Feasibility of laparoscopic management of acute haemoperitoneum secondary to ruptured ovarian cysts in a haemodynamically unstable patient. Minim Invasive Ther Allied Technol. 2011;20(1):46-49.

11. Terzic M, Likic I, Pilic I, Bila J, Knezevic N. Conservative management of massive hematoperitoneum caused by ovulation in a patient with severe form of von Willebrand disease--a case report. Clin Exp Obstet Gynecol. 2012;39(4):537-540. 
12. Hallatt JG, Steele CH, Snyder M. Ruptured corpus luteum with hemoperitoneum: A study of 173 surgical cases. Am J Obstet Gynecol. 1984;149(1):5-9.

13. Waters CH. HEMORRHAGE INTO AN OVARIAN CYST SIMULATING ECTOPIC PREGNANCY. JAMA J Am Med Assoc. 1918;70(5):295.

14. Phaneuf LE. INTRAPERITONEAL HEMORRHAGE FROM RUPTURED OVARIAN CYST. JAMA J Am Med Assoc. 1924;83(9):658.

15. Yoffe N, Bronshtein M, Brandes J, Blumenfeld Z. Hemorrhagic ovarian cyst detection by transvaginal sonography: The great imitator. Gynecol Endocrinol. 1991;5(2):123-129.

16. Roche O, Chavan N, Aquilina J, Rockall A. Radiological appearances of gynaecological emergencies. Insights Imaging. 2012;3(3):265-275.

17. Kim JH, Lee SM, Lee J-H, Jo YR, Moon MH, Shin J, et al. Successful Conservative Management of Ruptured Ovarian Cysts with Hemoperitoneum in Healthy Women. PLoS ONE. 2014;9(3):e91171.

18. Teng S-W, Tseng J-Y, Chang C-K, Li C-T, Chen Y-J, Wang P-H. Comparison of Laparoscopy and Laparotomy in Managing Hemodynamically Stable Patients with Ruptured Corpus Luteum with Hemoperitoneum. J Am Assoc Gynecol Laparosc. 2003;10(4):474-477. 